\title{
BLOW UP OF FRACTIONAL SCHRÖDINGER EQUATIONS ON MANIFOLDS WITH NONNEGATIVE RICCI CURVATURE
}

\author{
HUALI ZHANG AND SHILIANG ZHAO
}

\begin{abstract}
In this paper, the well-posedness of Cauchy's problem of fractional Schrödinger equations with a power type nonlinearity on $n$-dimensional manifolds with nonnegative Ricci curvature is studied. Under suitable volume conditions, the local solution with initial data in $H^{\left[\frac{n}{2}\right]+1}$ will blow up in finite time no matter how small the initial data is, which follows from a new weight function and ODE inequalities. Moreover, the upper-bound of the lifespan can be estimated.
\end{abstract}

\section{Introduction and Main results}

Let $0<\alpha<2$ and in this short article we study the Cauchy's problem of nonlinear fractional Schrödinger equations

$$
\left\{\begin{array}{l}
\mathrm{i} \partial_{t} u-(-\Delta)^{\frac{\alpha}{2}} u=F(u, \bar{u}),(t, x) \in(0,+\infty) \times M \\
\left.u\right|_{t=0}=\varphi_{0}(x)+\mathrm{i} \varphi_{1}(x),
\end{array}\right.
$$

where $\varphi_{0}(x), \varphi_{1}(x) \in C_{c}^{\infty}(M)$ are real valued functions and $M$ is a complete manifold with nonnegative Ricci curvature. $F$ is a nonlinear function of $u, \bar{u}$, satisfying

$$
|F(u, \bar{u})| \lesssim|u|^{p}
$$

where $\bar{u}$ is the conjugate of $u$ and $p>1$. Note that when $\alpha=2$, (1.1) is the nonlinear Schrödinger equation. When $1<\alpha<2$, (1.1) was introduced by Laskin in 37. Namely, the quantum mechanics path integral over Brownian trajectories leads to the well known Schrödinger equation $(\alpha=2)$, and the path integral over Lévy trajectories leads to the fractional Schrödinger equation $(1<\alpha<2)$.

First we recall some known results about the nonlinear Schrödinger equations on $\mathbb{R}^{n}$. The global existence results for small data can be established when $p$ is large enough. If $p>\frac{\sqrt{n^{2}+12 n+4}+n+2}{2 n}$, it was shown by Strauss [48, that the global solutions exist for small initial data. For $F=|u|^{p-1} u$, the wave operators can be constructed in general for small data when $p>1+\frac{2}{n}$ [18, 42. And hence the global existence is guaranteed by the conservation of mass and energy and the wave operators. When $p$ is small, the structure of the nonlinearity starts to play a crucial role. For example, consider $F=|u|^{p-1} u$ and $1<p<1+\frac{2}{n}$. For $1 \leq n \leq 3$, asymptotically free solutions can not exist [28, 43. For $F \stackrel{n}{=}|u|^{p}$, Ikeda-Inui 31] proved a small data blow-up result of $L^{2}$ when $1<p<1+\frac{4}{n}$. For other nonlinearities such as $u^{2}, \bar{u}^{2}$, few examples of global existence for small data

Date: April 2, 2020.

2010 Mathematics Subject Classification. Primary 35A01.

Key words and phrases. Fractional Schrödinger equations, blow-up, weight function, heat kernel. 
below the Strauss exponent are known. We refer the readers to [12, 20, 21, 25, 29, 35, 41 and references therein. Furthermore, there are some Strichartz estimates for nonlinear Schrödinger equations on manifolds, for instances [1, 4, 6, 3, 2, 5, 11, 9, 10, 14, 46, 32, 33.

As for nonlinear fractional Schrödinger equations, the Strichartz estimates was established by Cho-Koh-Seo 13 in the radial case. For $F=|u|^{p-1} u$, Guo-SireWang-Zhao 27] showed the global well-posedness of radial solutions in the energy critical case. Boulenger-Himmelsbach-Lenzmann [6] derived a blow-up result with radial data for (1.1) in both $L^{2}$-supercritical and $L^{2}$-critical cases respectively, and Guo-Zhu [26] further completed the blow-up result with radial data for general dimensions and nonlinearities. For cubic nonlinearity $|u|^{2} u$, Guo-Han-Xin 24] showed that the period boundary value problem of (1.1) is globally well-posed. IonescuPusateri 34] proved that the global small, smooth solutions of (1.1) exists if $\alpha=\frac{1}{2}$. Guo-Huo [25] established the global well-posedness of (1.1) if $1<\alpha<2$, where the key tri-linear estimates in Bourgain space played a important role. Concerning to blow-up of solutions for fractional Schrödinger equations on $\mathbb{R}^{n}$, there are some interesting results. For $\alpha=1$, Fujiwara in [15] proved that there is no global weak solution; Fino-Dannawi-Kirane in [16, 17 considered the blow up of mild solutions for $0<\alpha<2$. Motivated by these work, we are interested in blow up of strong solutions of general fractional Schrödinger equations on Riemannian manifolds.

In this paper, we get the blow-up results for a class of Schrödinger equations on Riemannian manifolds with nonnegative Ricci curvature. To be precise, let $(M, g)$ be a complete manifold of dimension $n$ with nonnegative Ricci curvature. Denote by $d$ the geodesic distance and $\mu$ the Riemannian measure. By the Bishop-Gromov inequality, $M$ satisfies the doubling condition: there exists constant $C>0$ such that

$$
V(x, 2 r) \leq C V(x, r), \quad \forall r>0, x \in M,
$$

where $V(x, r)$ is the volume of the geodesic ball centered at $x$ with radius $r$. In this paper, we will use the Einstein's summation convention. Then in local coordinates, the Laplace-Beltrami operator can be expressed as

$$
\Delta=\frac{1}{\sqrt{\operatorname{det} g}} \frac{\partial}{\partial x_{j}}\left(\sqrt{\operatorname{det} g} g^{j k} \frac{\partial}{\partial x_{k}}\right)
$$

where $\left(g^{j k}\right)_{1 \leq j, k \leq n}$ is the inverse matrix of $\left(g_{j k}\right)_{1 \leq j, k \leq n}$. For any $p \in M$, consider the normal coordinates in which the Riemannian metric can be written as

$$
g=d r^{2}+r^{2} g_{\alpha \beta}(r, \theta) d \theta^{\alpha} d \theta^{\beta} .
$$

Thus for any distance function $f(r)=f(d(x, p))$, we have by (1.3)

$$
\Delta f(r)=f^{\prime \prime}(r)+\frac{n-1}{r} f^{\prime}(r)+\frac{1}{\sqrt{G}} \frac{\partial \sqrt{G}}{\partial r} f^{\prime}(r)
$$

where $G=\operatorname{det}\left(g_{\alpha, \beta}\right)$. Denote by $p_{t}(x, y)$ the Schwartz kernel of the heat semigroup $e^{t \Delta}$. According to 39, the heat kernel $p_{t}(x, y)$ satisfies the Gaussian upper bound:

$$
p_{t}(x, y) \leq \frac{C}{V(x, \sqrt{t})} \exp \left(-c \frac{d^{2}(x, y)}{t}\right), \quad \forall t>0, x, y \in M .
$$

Then, by [23, the following estimates also hold by (1.5) and doubling properties

$$
\left|\Delta_{y} p_{t}(x, y)\right| \leq \frac{C}{V(x, \sqrt{t})} \frac{1}{t} \exp \left(-c \frac{d^{2}(x, y)}{t}\right), \quad \forall t>0, x, y \in M .
$$


Moreover, by 39](page 163 Theorem 1.3(i)), the following gradient estimates also hold under our assumption,

$$
\left|\nabla_{y} p_{t}(x, y)\right| \leq \frac{C}{V(x, \sqrt{t})} \frac{1}{\sqrt{t}} \exp \left(-c \frac{d^{2}(x, y)}{t}\right), \quad \forall t>0, x, y \in M .
$$

The main results of this paper is as follows:

Theorem 1.1. Let $0<\alpha<2, n \geq 2$ and $s=\left[\frac{n}{2}\right]+1$. Consider (1.1) on smooth complete $n$-dimensional manifolds $(M, g)$ with nonnegative Ricci curvature

$$
\left\{\begin{array}{l}
\mathrm{i} \partial_{t} u-(-\Delta)^{\frac{\alpha}{2}} u=F(u, \bar{u}) \\
u(0, x)=\varphi_{0}(x)+\mathrm{i} \varphi_{1}(x)
\end{array}\right.
$$

where $\varphi_{0}(x), \varphi_{1}(x) \in H^{s}(M)$. Assume that the following conditions holds

$$
\text { (1) }\left|\frac{r}{\sqrt{G}} \frac{\partial \sqrt{G}}{\partial r}\right| \leq C \quad \forall r>0 .
$$

$$
\text { (2) } V(x, r) \sim r^{n} \quad \forall x \in M, r>0 .
$$

If $\operatorname{Im} F=|u|^{p}, 1<p<1+\frac{\alpha}{n}$ and $\int_{M} \varphi_{0}(x) d \mu>0$, then the local solution of (1.1) will blow up in a finite time no matter how small the initial data is.

The proof of Theorem 1.1 crucially relies on constructing a new weight function and ODE inequalities. Different from Euclidian spaces, there is non-implicit formula for fractional Laplacian operator or heat kernel on general Riemannian manifolds. Hence, this work is not a trival extension from Euclidian spaces to manifolds with nonnegative Ricci curvature. By using Li-Yau's result [39, namely, some upper bounds of $\left|\nabla^{k} p_{t}\right|_{C^{0}}, k=0,1,2$ ( $p_{t}$ is the heat kernel), we could prove that local solutions with initial data in spaces $H^{\left[\frac{n}{2}\right]+1}(M)$ will blow up, where some ODE type estimates plays crucial role.

Remark 1.2. For all $p>1$, it's easy to see that the local solution of (1.1) exists in $H^{s}(M)\left(s=\left[\frac{n}{2}\right]+1\right)$ by bootstrap argument and contraction mapping principle. Applying $\nabla^{j}$ ( $j$ is a multi integer index) to (1.1) yields

$$
\partial_{t} \nabla^{j} u-(-\Delta)^{\frac{\alpha}{2}} \nabla^{j} u=\nabla^{j} F(u, \bar{u}) .
$$

Taking inner product with $\nabla^{j} \bar{u}$ on (1.1) and taking the imaginary part, and then summing it for $|j| \leq s$, we have

$$
\begin{aligned}
\|u(t, \cdot)\|_{H^{s}}^{2} & \lesssim\left\|u_{0}\right\|_{H^{s}}^{2}+\int_{0}^{t}\|u(\tau)\|_{H^{s}}^{2}\|u\|_{L^{\infty}}^{p-1} d \tau \\
& \lesssim\left\|u_{0}\right\|_{H^{s}}^{2}+\int_{0}^{t}\|u(\tau)\|_{H^{s}}^{p+1} d \tau,
\end{aligned}
$$

where in the last inequality we use that $H^{s} \hookrightarrow L^{\infty}$ for $s=\left[\frac{n}{2}\right]+1>\frac{n}{2}$. For sufficiently small $T>0$, we could obtain $u(t, x) \in C\left([0, T] ; H^{s}(M)\right)$. The uniqueness of solution follows from the similar energy estimates.

The paper is organized as follows: In Section 2, we prove some lemmas which play an important role in our proof; Our main results will be shown in Section 3; In Section 4, we give an example which satisfies our assumptions on the Riemannian metric. Now we introduce some notations. If $f, g$ are two functions, we say $f \lesssim g$ if and only if there exists a constant $c>0$ such that $f \leq c g$. We say $f \sim g$ if and only 
if there exits a constant $C>0$ such that $C^{-1} g \leq f \leq C g$. Set $f \wedge g=\min \{f, g\}$. The constant $C, c$ may change from line to line.

\section{Preliminary Results}

In this section, we will prove several lemmas which will be frequently used.

Lemma 2.1. Let $y>0$ and we have

$$
1 \wedge y \sim \frac{1}{1+y^{-1}}
$$

Proof. It is direct to check that

$$
\frac{1 \wedge y}{2} \leq \frac{1}{1+y^{-1}} \leq 1 \wedge y
$$

Lemma 2.2. Let $M$ be a n-dimensional complete Riemannian manifold with nonnegative Ricci curvature for $n \geq 2$. Set $\alpha, \gamma>0$ and the following hold:

(1) When $\gamma>\frac{n}{2}$, we have

$$
\int_{M}\left[t^{\frac{2}{\alpha}}+d^{2}(x, y)\right]^{-\gamma} d \mu(y) \lesssim t^{\frac{n-2 \gamma}{\alpha}}, \quad \forall \alpha, t, R>0, x \in M .
$$

(2) When $0 \leq \gamma<n$, we have

$$
\int_{B(x, R)} d(x, y)^{-\gamma} d \mu(y) \lesssim R^{n-\gamma}, \quad \forall \alpha, t, R>0, x \in M .
$$

(3) When $\gamma>n$, we have

$$
\int_{M \backslash B(x, R)} d(x, y)^{-\gamma} d \mu(y) \lesssim R^{n-\gamma}, \quad \forall \alpha, t, R>0, x \in M .
$$

Proof. The proof relies on the ring decomposition and facts that the volume of geodesic balls grow polynomially.

(1) When $\gamma>\frac{n}{2}$, we have by the ring decomposition

$$
\begin{aligned}
\int_{M}\left[t^{\frac{2}{\alpha}}+d^{2}(x, y)\right]^{-\gamma} d \mu(y) & =\sum_{j \in \mathbb{Z}} \int_{\left.B\left(x, 2^{j} t^{\frac{1}{\alpha}}\right) \backslash B\left(x, 2^{j-1} t^{\frac{1}{\alpha}}\right)^{\left[t^{\frac{2}{\alpha}}\right.}+d^{2}(x, y)\right]^{-\gamma} d \mu(y)} \\
& \lesssim \sum_{j \in \mathbb{Z}}\left[t^{\frac{2}{\alpha}}+2^{2 j-2} t^{\frac{2}{\alpha}}\right]^{-\gamma} 2^{j n} t^{\frac{n}{\alpha}} \\
& \lesssim t^{\frac{n-2 \gamma}{\alpha}} \sum_{j \in \mathbb{Z}}\left[1+2^{2 j-2}\right]^{-\gamma} 2^{j n}
\end{aligned}
$$

The above series converges as long as $\gamma>\frac{n}{2}$.

(2) For $0 \leq \gamma<n$, it is easy to check

$$
\begin{aligned}
\int_{B(x, R)} d(x, y)^{-\gamma} d \mu(y) & =\sum_{j \geq 0} \int_{B\left(x, 2^{-j} R\right) \backslash B\left(x, 2^{-j-1} R\right)} d(x, y)^{-\gamma} d \mu(y) \\
& \lesssim \sum_{j \geq 0} 2^{(j+1) \gamma} R^{-\gamma} 2^{-j n} R^{n} \lesssim R^{n-\gamma} .
\end{aligned}
$$


(3) Similarly we have for $\gamma>n$

$$
\begin{aligned}
\int_{M \backslash B(x, R)} d(x, y)^{-\gamma} d \mu(y) & =\sum_{j \geq 0} \int_{B\left(x, 2^{j+1} R\right) \backslash B\left(x, 2^{j} R\right)} d(x, y)^{-\gamma} d \mu(y) \\
& \lesssim \sum_{j \geq 0} 2^{-j \gamma} R^{-\gamma} 2^{(j+1) n} R^{n} \lesssim R^{n-\gamma} .
\end{aligned}
$$

The following estimates about the weight functions play an important role in our proof. Note that when $M=\mathbb{R}^{n}$, the following estimates are essentially known in 22, [40. Now we give the proof on manifolds.

Lemma 2.3. Let $M$ be a n-dimensional complete Riemannian manifold with nonnegative Ricci curvature for $n \geq 2$ and $h(t, x)=\frac{t^{1+\frac{n}{\alpha}}}{\left(d^{2}(x, p)+t^{\frac{2}{\alpha}}\right)^{\frac{n+\alpha}{2}}}$ for $x, p \in M$. For $0<\alpha<2$, we have

$$
\left|(-\Delta)^{\frac{\alpha}{2}} h(t, x)\right| \lesssim t^{-1} h(t, x), \quad \forall x \in M, t>0
$$

Proof. To start with, we have by [45](p.72, Theorem 6.9)

$$
\begin{aligned}
\left|(-\Delta)^{\frac{\alpha}{2}} h(t, x)\right| & =\frac{\sin \frac{\alpha}{2} \pi}{\pi} \int_{0}^{\infty} s^{\frac{\alpha}{2}-1}(-\Delta)(s I-\Delta)^{-1} h(t, x) d s \\
& =-\frac{\sin \frac{\alpha}{2} \pi}{\pi} \int_{0}^{\infty} s^{\frac{\alpha}{2}-1}(s I-\Delta)^{-1} \Delta h(t, x) d s \\
& =\int_{M} K(x, y) \Delta_{y} h(t, y) d \mu(y),
\end{aligned}
$$

where $K(x, y)$ is the integral kernel of $-\frac{\sin \frac{\alpha}{2} \pi}{\pi} \int_{0}^{\infty} s^{\frac{\alpha}{2}-1}(s I-\Delta)^{-1} d s$ By Lemma 2.1 we have

$$
\frac{t^{1+\frac{n}{\alpha}}}{\left[d(x, p)^{2}+t^{\frac{2}{\alpha}}\right]^{\frac{n+\alpha}{2}}}=\frac{1}{\left[1+\left(\frac{d(x, p)}{t^{\frac{1}{\alpha}}}\right)^{2}\right]^{\frac{n+\alpha}{2}}} \sim\left[1 \wedge\left(\frac{t^{\frac{1}{\alpha}}}{d(x, p)}\right)^{2}\right]^{\frac{n+\alpha}{2}} .
$$

Thus it is sufficient to prove

$$
\left|\int_{M} K(x, y) \Delta_{y} h(t, y) d \mu(y)\right| \lesssim t^{-1}\left[1 \wedge\left(\frac{t^{\frac{1}{\alpha}}}{d(x, p)}\right)^{2}\right]^{\frac{n+\alpha}{2}} .
$$

Indeed, we have to distinguish two cases.

Case I: $d(x, p) \leq t^{\frac{1}{\alpha}}$.

Note that in local coordinate, we have by (1.4)

$$
\Delta h(t, y)=\frac{\partial^{2} h(t, r)}{\partial r^{2}}+\frac{n-1}{r} \frac{\partial h(t, r)}{\partial r}+\frac{1}{\sqrt{G}} \frac{\partial \sqrt{G}}{\partial r} \frac{\partial h(t, r)}{\partial r}
$$

where $r=d(y, p)$. According to the definition of $h$, we obtain

$$
\begin{aligned}
& \frac{\partial h(t, r)}{\partial r}=-\frac{(n+\alpha) t^{1+\frac{n}{\alpha}} r}{\left[t^{\frac{2}{\alpha}}+r^{2}\right]^{\frac{n+\alpha}{2}+1}}, \\
& \frac{\partial^{2} h(t, r)}{\partial r^{2}}=-\frac{(n+\alpha) t^{1+\frac{n}{\alpha}}}{\left[t^{\frac{2}{\alpha}}+r^{2}\right]^{\frac{n+\alpha}{2}}+1}+\frac{(n+\alpha)(n+\alpha+2) t^{1+\frac{n}{\alpha}} r}{\left[t^{\frac{2}{\alpha}}+r^{2}\right]^{\frac{n+\alpha}{2}+2}} .
\end{aligned}
$$


In turn, we have by (1.8)

$$
|\Delta h(t, y)| \lesssim \frac{t^{1+\frac{n}{\alpha}}}{\left[t^{\frac{2}{\alpha}}+r^{2}\right]^{\frac{n+\alpha}{2}+1}} .
$$

Moreover, by [45](p.8) and (1.5), we obtain

$$
\begin{aligned}
\left|\int_{M} K(x, y) \psi(y) d \mu(y)\right| & =c\left|\int_{0}^{\infty} s^{\frac{\alpha}{2}-1}(s I-\Delta)^{-1} \psi d s\right| \\
& =c\left|\int_{0}^{\infty} s^{\frac{\alpha}{2}-1} \int_{0}^{\infty} e^{-s \tau} e^{\tau \Delta} \psi d \tau d s\right| \\
& =c^{\prime}\left|\int_{0}^{\infty} e^{\tau \Delta} \psi \tau^{-\frac{\alpha}{2}} d \tau\right| \\
& \lesssim \int_{0}^{\infty} \int_{M} \frac{\tau^{-\frac{\alpha}{2}}}{V(x, \sqrt{\tau})} \exp \left(-c \frac{d^{2}(x, y)}{\tau}\right)|\psi(y)| d \mu(y) d \tau \\
& \lesssim \int_{M} d(x, y)^{2-n-\alpha}|\psi(y)| d \mu(y) .
\end{aligned}
$$

As a result, it follows

$$
\left|\int_{M} K(x, y) \Delta_{y} h(t, y) d \mu(y)\right| \lesssim \int_{M} \frac{t^{1+\frac{n}{\alpha}}}{\left[t^{\frac{2}{\alpha}}+d(y, p)^{2}\right]^{\frac{n+\alpha}{2}}+1} d(x, y)^{2-n-\alpha} d \mu(y) \triangleq I .
$$

Set

$$
I=\int_{d(x, y) \leq t^{\frac{1}{\alpha}}}+\int_{d(x, y)>t^{\frac{1}{\alpha}}} \triangleq I_{1}+I_{2} .
$$

For $I_{1}$, we have by Lemma 2.2

$$
I_{1} \lesssim t^{1+\frac{n}{\alpha}-\frac{2}{\alpha}\left(\frac{n+\alpha}{2}+1\right)} \int_{d(x, y) \leq t^{\frac{1}{\alpha}}} d(x, y)^{2-n-\alpha} d \mu(y) \lesssim t^{-1} .
$$

Note that we have used the assumption $0<\alpha<2$.

For $I_{2}$, we obtain

$$
\begin{aligned}
I_{2} & \lesssim t^{1+\frac{n}{\alpha}+\frac{1}{\alpha}(2-n-\alpha)} \int_{d(x, y)>t^{\frac{1}{\alpha}}}\left[t^{\frac{2}{\alpha}}+d(y, p)^{2}\right]^{-\frac{n+\alpha}{2}-1} d \mu(y) \\
& \lesssim t^{\frac{2}{\alpha}} \int_{M}\left[t^{\frac{2}{\alpha}}+d(y, p)^{2}\right]^{-\frac{n+\alpha}{2}-1} d \mu(y) \lesssim t^{-1} .
\end{aligned}
$$

We have used Lemma 2.2 in the last step. Thus (2.1) holds for $d(x, p) \leq t^{\frac{1}{\alpha}}$.

Case II: $d(x, p)>t^{\frac{1}{\alpha}}$.

Let $\varphi_{1}(t), \varphi_{2}(t), \varphi_{3}(t)$ be the partition of unity, i.e.,

$$
\varphi_{1}(t)+\varphi_{2}(t)+\varphi_{3}(t)=1, \quad \text { and } \quad 0 \leq \varphi_{k}(t) \leq 1 \quad \forall t>0, k=1,2,3 .
$$

$\varphi_{1}$ is supported in $0<t<\frac{3}{4} d(x, p), \varphi_{2}$ is supported in $\frac{1}{2} d(x, p)<t<2 d(x, p)$ and equals 1 for $\frac{3}{4} d(x, p) \leq t \leq \frac{5}{4} d(x, p), \varphi_{1}$ is supported in $\frac{5}{4} d(x, p)<t$. Moreover, they are smooth functions. For more properties of partition of unity we refer the readers to 30 (p.25).

Set for $k=1,2,3$

$$
\Pi_{k}=\int_{M} K(x, y) \varphi_{k}(d(x, y)) \Delta_{y} h(t, y) d \mu(y) .
$$


For $\Pi_{1}$, by Lemma 2.2 we obtain

$$
\begin{aligned}
\left|\Pi_{1}\right| & \lesssim \int_{M} d(x, y)^{2-n-\alpha}\left|\Delta_{y} h(t, y)\right| d \mu(y) \\
& \lesssim t^{1+\frac{n}{\alpha}}\left[t^{\frac{2}{\alpha}}+d(x, p)^{2}\right]^{-\frac{n+\alpha}{2}-1} \int_{d(x, y) \leq \frac{3 d(x, p)}{4}} d(x, y)^{2-n-\alpha} d \mu(y) \\
& \lesssim t^{1+\frac{n}{\alpha}} d(x, p)^{-n-2 \alpha} \lesssim \frac{t^{\frac{n}{\alpha}}}{d(x, p)^{n+\alpha}} .
\end{aligned}
$$

We have used the fact $d(y, p) \geq d(x, p)-d(x, y) \geq \frac{1}{4} d(x, p)$ in the first inequality. For $\Pi_{3}$, we have

$$
\begin{aligned}
\left|\Pi_{3}\right| & \lesssim t^{1+\frac{n}{\alpha}} \int_{d(x, y)>\frac{5 d(x, p)}{4}}\left[t^{\frac{2}{\alpha}}+d(y, p)^{2}\right]^{-\frac{n+\alpha}{2}-1} d(x, y)^{2-n-\alpha} d \mu(y) \\
& \lesssim t^{1+\frac{n}{\alpha}} d(x, p)^{-n-\alpha} \int_{d(x, y)>\frac{5 d(x, p)}{4}}\left[t^{\frac{2}{\alpha}}+d(y, p)^{2}\right]^{-\frac{n+\alpha}{2}-1} d(x, y)^{2} d \mu(y) \\
& \lesssim t^{1+\frac{n}{\alpha}} d(x, p)^{-n-\alpha}\left(J_{1}+J_{2}\right),
\end{aligned}
$$

where

$$
\begin{aligned}
& J_{1}=\int_{d(y, p) \leq 2 d(x, p) \cap d(x, y)>\frac{5 d(x, p)}{4}}\left[t^{\frac{2}{\alpha}}+d(y, p)^{2}\right]^{-\frac{n+\alpha}{2}-1} d(x, y)^{2} d \mu(y), \\
& J_{2}=\int_{d(y, p)>2 d(x, p) \cap d(x, y)>\frac{5 d(x, p)}{4}}\left[t^{\frac{2}{\alpha}}+d(y, p)^{2}\right]^{-\frac{n+\alpha}{2}-1} d(x, y)^{2} d \mu(y) .
\end{aligned}
$$

Note that for $J_{1}$ the following holds

$$
\begin{aligned}
J_{1} & \lesssim d(x, p)^{2} \int_{B(p, 2 d(x, p))} d(x, p)^{-n-\alpha-2} d \mu(y) \\
& \lesssim d(x, p)^{-\alpha}
\end{aligned}
$$

where we have used the facts $d(x, y) \leq d(x, p)+d(y, p) \leq 3 d(x, p)$ and $d(y, p) \geq$ $d(x, y)-d(x, p) \geq \frac{1}{4} d(x, p)$.

Moreover

$$
\begin{aligned}
J_{2} & \lesssim \int_{M \backslash B(p, 2 d(x, p))} d(y, p)^{-n-\alpha-2} d(y, p)^{2} d \mu(y) \\
& \lesssim d(x, p)^{-\alpha} .
\end{aligned}
$$

We have used the facts $d(x, y) \leq d(x, p)+d(y, p) \leq \frac{3}{2} d(y, p)$. As a result, we obtain

$$
\left|\Pi_{3}\right| \lesssim \frac{t^{\frac{n}{\alpha}}}{d(x, p)^{n+\alpha}}
$$

Now we are ready to deal with $\Pi_{2}$. To this end we need the integration by parts.

$$
\begin{aligned}
\Pi_{2}= & \int_{M} K(x, y) \varphi_{2}(d(x, y)) \Delta_{y} h(t, y) d \mu(y) \\
= & \int_{M} \Delta_{y}\left[K(x, y) \varphi_{2}(d(x, y))\right] h(t, y) d \mu(y) \\
= & \int_{M}\left[\Delta_{y} K(x, y) \varphi_{2}(d(x, y))+2\left\langle\nabla_{y} K(x, y), \nabla_{y} \varphi_{2}(d(x, y))\right\rangle_{g}\right. \\
& \left.+K(x, y) \Delta_{y} \varphi_{2}(d(x, y))\right] h(t, y) d \mu(y)
\end{aligned}
$$


where $\langle\cdot\rangle_{g}$ is the Riemannian metric on the tangent spaces.

As above, we have by (1.6)

$$
\begin{aligned}
& \left|\int_{M} \Delta_{y} K(x, y) h(t, y) d \mu(y)\right| \\
= & c\left|\int_{0}^{\infty} \int_{M} \tau^{-\frac{\alpha}{2}} \Delta_{y} p_{\tau}(x, y) h(t, y) d \mu(y) d \tau\right| \\
\lesssim & \int_{M} \int_{0}^{\infty} \frac{\tau^{-\frac{\alpha}{2}-1}}{V(x, \sqrt{\tau})} \exp \left(-c \frac{d^{2}(x, y)}{\tau}\right) d \tau|h(t, y)| d \mu(y) \\
\lesssim & \int_{M} d(x, y)^{-n-\alpha}|h(t, y)| d \mu(y) .
\end{aligned}
$$

It follows that

$$
\begin{aligned}
& \left|\int_{M}\left[\Delta_{y} K(x, y) \varphi_{2}(d(x, y))\right] h(t, y) d \mu(y)\right| \\
& \lesssim t^{1+\frac{n}{\alpha}} \int_{\frac{d(x, p)}{2} \leq d(x, y) \leq 2 d(x, p)}\left[t^{\frac{2}{\alpha}}+d(y, p)^{2}\right]^{-\frac{n+\alpha}{2}} d(x, y)^{-n-\alpha} d \mu(y) \\
& \lesssim t^{1+\frac{n}{\alpha}} d(x, p)^{-n-\alpha} \int_{\frac{d(x, p)}{2} \leq d(x, y) \leq 2 d(x, p)}\left[t^{\frac{2}{\alpha}}+d(y, p)^{2}\right]^{-\frac{n+\alpha}{2}} d \mu(y) \\
& \lesssim t^{1+\frac{n}{\alpha}} d(x, p)^{-n-\alpha}\left(\int_{E_{1}}\left[t^{\frac{2}{\alpha}}+d(y, p)^{2}\right]^{-\frac{n+\alpha}{2}} d \mu(y)+\int_{E_{2}}\left[t^{\frac{2}{\alpha}}+d(y, p)^{2}\right]^{-\frac{n+\alpha}{2}} d \mu(y)\right)
\end{aligned}
$$

where $E_{1}=B\left(p, \frac{1}{2} d(x, p)\right)$ and $E_{2}=\left\{y \mid \frac{d(x, p)}{2} \leq d(x, y) \leq 2 d(x, p)\right\} \backslash B\left(p, \frac{1}{2} d(x, p)\right)$. Note that

$$
\begin{aligned}
& \int_{E_{1}}\left[t^{\frac{2}{\alpha}}+d(y, p)^{2}\right]^{-\frac{n+\alpha}{2}} d \mu(y) \\
\lesssim & \int_{d(y, p) \leq \frac{t}{\alpha}}\left[t^{\frac{2}{\alpha}}+d(y, p)^{2}\right]^{-\frac{n+\alpha}{2}} d \mu(y)+\int_{\frac{t}{\alpha}} \leq d(y, p) \leq \frac{d(x, p)}{2} \\
\lesssim & t^{-1}+\int_{\frac{\frac{1}{\alpha}}{2} \leq d(y, p)}\left[t^{\frac{2}{\alpha}}+d(y, p)^{2}\right]^{-\frac{n+\alpha}{2}} d \mu(y) \lesssim t^{-1} .
\end{aligned}
$$

On the other hand, since $d(y, p) \geq \frac{1}{2} d(x, p)$ for $y \in E_{2}$, we have

$$
\int_{E_{1}}\left[t^{\frac{2}{\alpha}}+d(y, p)^{2}\right]^{-\frac{n+\alpha}{2}} d \mu(y) \lesssim d(x, p)^{-n-\alpha} V(x, 2 d(x, p)) \lesssim d(x, p)^{-\alpha}
$$

As a result, we conclude

$$
\left|\int_{M}\left[\Delta_{y} K(x, y) \varphi_{2}(d(x, y))\right] h(t, y) d \mu(y)\right| \lesssim \frac{t^{\frac{n}{\alpha}}}{d(x, p)^{n+\alpha}}
$$


To proceed we need the following estimates:

$$
\begin{aligned}
& \left|\int_{M}\left\langle\nabla_{y} K(x, y), \nabla_{y} \varphi_{2}(d(x, y))\right\rangle_{g} h(t, y) d \mu(y)\right| \\
= & c\left|\int_{0}^{\infty} \int_{M} \tau^{-\frac{\alpha}{2}}\left\langle\nabla_{y} p_{\tau}(x, y), \nabla_{y} \varphi_{2}(d(x, y))\right\rangle_{g} h(t, y) d \mu(y) d \tau\right| \\
\lesssim & \int_{E_{3}} \int_{0}^{\infty} \frac{\tau^{-\frac{\alpha}{2}-\frac{1}{2}}}{V(x, \sqrt{\tau})} \exp \left(-c \frac{d^{2}(x, y)}{\tau}\right) d \tau d(x, p)^{-1}|h(t, y)| d \mu(y) \\
\lesssim & d(x, p)^{-1} \int_{E_{3}} d(x, y)^{1-n-\alpha}|h(t, y)| d \mu(y)
\end{aligned}
$$

where $E_{3}=\frac{d(x, p)}{2} \leq d(x, y) \leq \frac{3 d(x, p)}{4} \cup \frac{5 d(x, p)}{4} \leq d(x, y) \leq 2 d(x, p)$. We have used assumption (1.7) and the facts $\left|\varphi_{2}^{\prime}(d(x, y))\right| \leq c d(x, p)^{-1}$. See for example [30] for the properties of cut-off functions.

Then we have

$$
\begin{aligned}
& \left|\int_{M}\left\langle\nabla_{y} K(x, y), \nabla_{y} \varphi_{2}(d(x, y))\right\rangle_{g} h(t, y) d \mu(y)\right| \\
\lesssim & t^{1+\frac{n}{\alpha}} d(x, p)^{-1} \int_{E_{3}}\left[t^{\frac{2}{\alpha}}+d(y, p)^{2}\right]^{-\frac{n+\alpha}{2}} d(x, y)^{1-n-\alpha} d \mu(y) \\
\lesssim & t^{1+\frac{n}{\alpha}} d(x, p)^{-(n+\alpha)} \int_{E_{3}}\left[t^{\frac{2}{\alpha}}+d(y, p)^{2}\right]^{-\frac{n+\alpha}{2}} d \mu(y) \\
\lesssim & t^{1+\frac{n}{\alpha}} d(x, p)^{-(n+\alpha)} \cdot t^{-1} \\
\lesssim & \frac{t^{\frac{n}{\alpha}}}{d(x, p)^{n+\alpha}} .
\end{aligned}
$$

Similarly we have

$$
\begin{aligned}
& \left|\int_{M} K(x, y) \Delta_{y} \varphi_{2}(d(x, y)) h(t, y) d \mu(y)\right| \\
= & c\left|\int_{0}^{\infty} \int_{M} \tau^{-\frac{\alpha}{2}} p_{\tau}(x, y) \Delta_{y} \varphi_{2}(d(x, y)) h(t, y) d \mu(y) d \tau\right| \\
\lesssim & \int_{E_{3}} \int_{0}^{\infty} \frac{\tau^{-\frac{\alpha}{2}}}{V(x, \sqrt{\tau})} \exp \left(-c \frac{d^{2}(x, y)}{\tau}\right) d \tau d(x, p)^{-2}|h(t, y)| d \mu(y) \\
\lesssim & d(x, p)^{-2} \int_{E_{3}} d(x, y)^{2-n-\alpha}|h(t, y)| d \mu(y) .
\end{aligned}
$$

$E_{3}$ is as above and we have used the facts that in local coordinates

$$
\Delta_{y} \varphi_{2}(r)=\varphi_{2}^{\prime \prime}(r)+\frac{n-1}{r} \varphi_{2}^{\prime}(r)+\frac{1}{\sqrt{G}} \frac{\partial \sqrt{G}}{\partial r} \varphi_{2}^{\prime}(r)
$$


where $r=d(x, y)$. Hence we have $\left|\Delta_{y} \varphi_{2}(r)\right| \leq c d(x, p)^{-2}$ for $y \in E_{3}$. Thus we obtain

$$
\begin{aligned}
& \left|\int_{M} K(x, y) \Delta_{y} \varphi_{2}(d(x, y)) h(t, y) d \mu(y)\right| \\
\lesssim & t^{1+\frac{n}{\alpha}} d(x, p)^{-2} \int_{E_{3}}\left[t^{\frac{2}{\alpha}}+d(y, p)^{2}\right]^{-\frac{n+\alpha}{2}} d(x, y)^{2-n-\alpha} d \mu(y) \\
\lesssim & t^{1+\frac{n}{\alpha}} d(x, p)^{-(n+\alpha)} \int_{E_{3}}\left[t^{\frac{2}{\alpha}}+d(y, p)^{2}\right]^{-\frac{n+\alpha}{2}} d \mu(y) \\
\lesssim & t^{1+\frac{n}{\alpha}} d(x, p)^{-(n+\alpha)} t^{-1} \\
\lesssim & \frac{t^{\frac{n}{\alpha}}}{d(x, p)^{n+\alpha}} .
\end{aligned}
$$

As a result, we have proved

$$
\left|\Pi_{2}\right| \lesssim \frac{t^{\frac{n}{\alpha}}}{d(x, p)^{n+\alpha}}
$$

Combining these estimates, we have proved the desired results.

\section{Proof of Theorem 1.1}

For each $\phi_{0}, \phi_{1} \in H^{\left[\frac{n}{2}\right]+1}$, there is a positive number $T_{0}$ and a unique solution $u \in C\left(\left[0, T_{0}\right] ; H^{\left[\frac{n}{2}\right]+1}\right)$ for Cauchy's problem (1.1). Let

$$
u(t, x)=w(t, x)+\mathrm{i} v(t, x),
$$

and take the imaginary part of the nonlinear fractional Schrödinger equation (1.1), we have

$$
\left\{\begin{array}{l}
w_{t}-(-\Delta)^{\frac{\alpha}{2}} v=\left(w^{2}+v^{2}\right)^{\frac{p}{2}} \\
t=0: w=\varphi_{0}(x)
\end{array}\right.
$$

Now fix a point $p \in M$. For $0<\alpha<2$, let $h(t, x)=\frac{t^{1+\frac{n}{\alpha}}}{\left(d^{2}(x, p)+t^{\frac{2}{\alpha}}\right)^{\frac{n+\alpha}{2}}}$ and consider the following function,

$$
\phi(t)=\int_{M} h(T, x) w(t, x) d \mu(x),
$$

where $T=t+N$ and $N$ will be determined later. To start with, we have

$$
\begin{aligned}
\phi^{\prime}(t) & =\int_{M} h(T, x) w_{t} d \mu(x)+\int_{M} \partial_{t} h(T, x) w d \mu(x) \\
& =\int_{M} h(T, x)\left((-\Delta)^{\frac{\alpha}{2}} v+\left(w^{2}+v^{2}\right)^{\frac{p}{2}}\right) d \mu(x)+\int_{M} \partial_{t} h(T, x) w d \mu(x) .
\end{aligned}
$$

Note that $(-\Delta)^{\frac{\alpha}{2}}$ is self-adjoint. It follows

$$
\phi^{\prime}(t)=\int_{M} \partial_{t} h(T, x) w+(-\Delta)^{\frac{\alpha}{2}} h(T, x) v d \mu(x)+\int_{M} h(T, x)\left(w^{2}+v^{2}\right)^{\frac{p}{2}} d \mu(x) .
$$

Now consider

$$
I=\int_{M} \partial_{t} h(T, x) w+(-\Delta)^{\frac{\alpha}{2}} h(T, x) v d \mu(x) .
$$


Note that

$$
\begin{aligned}
\left|\partial_{t} h(T, x)\right| & =\left|\left(1+\frac{n}{\alpha}\right) \frac{T^{\frac{n}{\alpha}}}{\left(d^{2}(x, p)+T^{\frac{2}{\alpha}}\right)^{\frac{\alpha+n}{2}}}-\left(1+\frac{n}{\alpha}\right) \frac{T^{\frac{n+2}{\alpha}}}{\left(d^{2}(x, p)+T^{\frac{2}{\alpha}}\right)^{\frac{\alpha+n}{2}+1}}\right| \\
& \lesssim \frac{1}{T} h(T, x) .
\end{aligned}
$$

By Lemma 2.3, we have

$$
\begin{aligned}
|I| & \lesssim \int_{M} T^{-1} h(T, x)(|w|+|v|) d \mu(x) \\
& \lesssim T^{-1}\left(\int_{M} h(T, x)\left(w^{2}+v^{2}\right)^{\frac{p}{2}} d \mu(x)\right)^{\frac{1}{p}}\left(\int_{M} h(T, x) d \mu(x)\right)^{\frac{1}{p^{\prime}}} \\
& \lesssim T^{\frac{n}{\alpha p^{\prime}}-1}\left(\int_{M} h(T, x)\left(w^{2}+v^{2}\right)^{\frac{p}{2}} d \mu(x)\right)^{\frac{1}{p}}
\end{aligned}
$$

where we have used the Hölder inequality in the second step. As a result, we have

$$
|I| \leq \frac{1}{2} \int_{M} h(T, x)\left(w^{2}+v^{2}\right)^{\frac{p}{2}} d \mu(x)+C T^{\frac{n}{\alpha}-p^{\prime}} .
$$

In turn, we obtain

$$
\phi^{\prime}(t) \geq \frac{1}{2} \int_{M} h(T, x)\left(w^{2}+v^{2}\right)^{\frac{p}{2}} d \mu(x)-C T^{\frac{n}{\alpha}-p^{\prime}} .
$$

On the other hand,

$$
\begin{aligned}
|\phi(t)| & \leq\left(\int_{M} h(T, x)|w|^{p} d \mu(x)\right)^{\frac{1}{p}}\left(\int_{M} h(T, x) d \mu(x)\right)^{\frac{1}{p^{\prime}}} \\
& \lesssim T^{\frac{n}{\alpha p^{\prime}}}\left(\int_{M} h(T, x)\left(w^{2}+v^{2}\right)^{\frac{p}{2}} d \mu(x)\right)^{\frac{1}{p}} .
\end{aligned}
$$

Then we obtain

$$
\phi^{\prime}(t) \geq C \frac{|\phi(t)|^{p}}{T^{\frac{n}{\alpha}(p-1)}}-C T^{\frac{n}{\alpha}-p^{\prime}}
$$

Therefore we have

$$
\phi(t) \geq \int_{M} h(N, x) \varphi_{0}(x) d \mu(x)-C N^{\frac{n}{\alpha}-p^{\prime}+1}+C \int_{0}^{t} \frac{|\phi(\tau)|^{p}}{(\tau+N)^{\frac{n}{\alpha}(p-1)}} d \tau .
$$

Note that $\frac{n}{\alpha}-p^{\prime}+1<0$ whenever $p<1+\frac{\alpha}{n}$. By the dominated convergence theorem, the following holds,

$$
\lim _{N \rightarrow \infty} \int_{M} h(N, x) \varphi_{0}(x) d \mu(x)=\int_{M} \varphi_{0}(x) d \mu(x) .
$$

Thus for $N$ large enough, we conclude that

$$
\phi(t) \geq \frac{1}{2} \int_{M} \varphi_{0}(x) d \mu(x)+C \int_{0}^{t} \frac{|\phi(\tau)|^{p}}{(\tau+N)^{\frac{n}{\alpha}(p-1)}} d \tau .
$$

Denote

$$
\varphi(t):=\frac{1}{2} \int_{M} \varphi_{0}(x) d \mu(x)+C \int_{0}^{t} \frac{|\phi(\tau)|^{p}}{(\tau+N)^{\frac{n}{\alpha}(p-1)}} d \tau .
$$


It follows

Finally we get

$$
\varphi^{\prime}(t) \geq C \frac{\varphi^{p}(t)}{(t+N)^{\frac{n}{\alpha}(p-1)}}
$$

$$
\varphi(t) \geq C\left[\varphi^{1-p}(0)+N^{1-\frac{n}{\alpha}(p-1)}-(t+N)^{1-\frac{n}{\alpha}(p-1)}\right]^{\frac{-1}{p-1}} .
$$

Set

$$
t_{*}=\left(N^{1-\frac{n}{\alpha}(p-1)}+\varphi(0)^{1-p}\right)^{\frac{1}{1-\frac{n}{\alpha}(p-1)}}-N .
$$

Hence,

$$
\varphi(t) \geq C\left(\left(t_{*}+N\right)^{1-\frac{n}{\alpha}(p-1)}-(t+N)^{1-\frac{n}{\alpha}(p-1)}\right)^{-\frac{1}{p-1}} .
$$

Since $1-\frac{n}{\alpha}(p-1)>0$ when $1<p<1+\frac{\alpha}{n}$, then

$$
\left(\left(t_{*}+N\right)^{1-\frac{n}{\alpha}(p-1)}-(t+N)^{1-\frac{n}{\alpha}(p-1)}\right)^{-\frac{1}{p-1}} \rightarrow+\infty, \text { if } t \rightarrow t_{*}^{-} .
$$

Therefore,

$$
\varphi(t) \rightarrow+\infty \text {, if } t \rightarrow t_{*}^{-} .
$$

Seeing from (3.3)-(3.4), then $\phi(t)$ tends to infinity if $t \rightarrow t_{*}$. A direct calculation tells us

$$
\begin{aligned}
\|h(T, x)\|_{L^{2}(M)} & \lesssim T^{1+\frac{n}{\alpha}}\left(\int_{M}\left((d(x, p))^{2}+T^{\frac{2}{\alpha}}\right)^{-(n+\alpha)} d \mu(x)\right)^{\frac{1}{2}} \\
& \lesssim T^{-1}
\end{aligned}
$$

By Hölder's inequality and (3.2), we derive that

$$
\phi(t) \leq\|h(T, \cdot)\|_{L^{2}(M)}\|w(t, \cdot)\|_{L^{2}(M)} .
$$

As a result,

$$
\|w(t, \cdot)\|_{L_{x}^{2}(M)} \geq T \phi(t) .
$$

Therefore, $\|w(t, \cdot)\|_{L_{x}^{2}(M)}$ tends to infinity if $t \rightarrow t_{*}$, and $T_{0}<t_{*}$. Hence we have proved the theorem.

\section{Appendix}

Note that when $M$ is a rotationally symmetric manifold with nonnegative Ricci curvature, (1.8) holds automatically. In fact, the Riemannian metric can be expressed as

$$
g=d r^{2}+\phi(r)^{2} \tilde{g}_{\alpha \beta}(\theta) d \theta^{\alpha} d \theta^{\beta}
$$

where $\phi(0)=0, \phi^{\prime}(0)=1, \phi(r)>0$ for every $r>0$ and $\tilde{g}$ is the standard metric on sphere. Thus we have $\sqrt{G}=\left(\frac{\phi(r)}{r}\right)^{n-1} \sqrt{\operatorname{det} \tilde{g}}$. According to the nonnegative of Ricci curvature, we have $\frac{\phi^{\prime \prime}(r)}{\phi(r)} \leq 0$ ( see $\S 3.2 .3$ of [4] $)$. Then the facts $\phi(r)>0$ and $\phi^{\prime}(0)=1$ give that $\phi^{\prime}(r)$ is a decreasing function with $0 \leq \phi^{\prime}(r) \leq 1$ for $r>0$. Thus we have

$$
\phi^{\prime}(r) \leq \frac{1}{r} \int_{0}^{r} \phi^{\prime}(s) d s=\frac{\phi(r)}{r} .
$$

It is direct to check that $\frac{r}{\sqrt{G}} \frac{\partial \sqrt{G}}{\partial r}=(n-1) \frac{r \phi^{\prime}(r)-\phi(r)}{\phi(r)}$ and hence (1.8) holds.

Note also that by our proof the range of $p$ in Theorem 1.1 is determined by the volume growth of geodesic balls on manifolds and $\frac{\alpha}{n} \rightarrow 0$ as $n \rightarrow \infty$. Thus it 
explains why the blow-up results of Schrödinger equations with polynomial nonlinearities on hyperbolic spaces should not be expected. As is known, hyperbolic spaces have the Ricci curvature -1 and the volume of balls grow exponentially.

\section{Acknowledgement}

The authors would like to thank the anonymous referee for his/her careful reading and valuable suggestions. The authors would like to express the gratitude to Prof. Yi Zhou for his helpful discussions and advice. The second author would also thank Prof. Changxing Miao and Prof. Jiqiang Zheng for valuable recommendations. The first author is supported by the State Scholarship Fund of China Scholarship Council ( No.201808430121); Hunan Provincial Key Laboratory of Intelligent Processing of Big Data on Transportation, Changsha University of Science and Technology, Changsha, 410114, China; and the Swedish Research Council under grant no. 2016-06596 while the second author was in residence at Institut MittagLeffler in Djursholm, Sweden during the year of 2019. She also thanks to Prof. Lars Andersson, for his hospital invitation and much help at Institut Mittag-Leffler. The second author is supported by the National Natural Science Foundation of China under Grant No.11901407.

\section{REFERENCES}

1. J. P. Anker, V. Pierfelice. Nonlinear Schrödinger equation on real hyperbolic spaces, Ann. Inst. H. Poincar Anal. NonLinéaire 2009; 26: 1853-1869.

2. V. Banica. The nonlinear Schrödinger equation on the hyperbolic space, Comm. Partial Differ. Equ. 2007; 32(10): 1643-1677.

3. V. Banica, R. Carles and G. Staffilani. Scattering theory for radial nonlinear Schrödinger equations on hyperbolic space, Geom. Funct. Anal. 2008; 18: 367-399.

4. V. Banica, T. Duyckaerts. Weihgted Strichartz estimates for radial Schrödinger equations on noncompact manifolds, Dyn. Partial Differ. Equ. 2007; 4: 335-359.

5. M. D. Blair, H. F. Smith, C. D. Sogge. Strichartz estimates and the nonlinear Schrödinger equation on manifolds with boundary, Math. Ann. 2012; 354: 1397-1430.

6. T. Boulenger, D. Himmelsbach and E. Lenzmann. Blowup for fractional NLS, J. Funct. Anal. 2016; 271: 2569-2603.

7. J. Bourgain. Fourier transform restriction phenomena for certain lattice subsets and applications to nonlinear evolution equations. I. Schrödinger equations, Geom. Funct. Anal. 1993; 3: $107-156$.

8. J. Bourgain. Exponential sums and nonlinear Schrödinger equations, Geom. Funct. Anal. 1993; 3: 157- 178.

9. N. Burq, P. Gérard and N. Tzvetkov. An instability property of the nonlinear Schrödinger equation on $\mathbb{S}^{d}$, Math. Res. Lett. 2002; 9: 323-335.

10. N. Burq, P. Gérard and N. Tzvetkov. Strichartz inequalities andnonlinear Schrödinger equation on on compact manifolds, Amer. J. Math. 2004; 126: 569-605.

11. N. Burq, P. Gérard and N. Tzvetkov. Bilinear eigenfunction estimates and the nonlinear Schrödinger equation on surfaces, Invent. Math. 2005; 159: 187-223.

12. T. Cazenave, F. Weissler. Rapidly decaying solutions of the nonlinear Schrödinger equation, Comm. Math. Phys. 1992; 147(1): 75-100.

13. C.H. Cho, Y. Koh and I. Seo. On inhomogeneous Strichartz estimates for fractional Schrödinger equations and their applications. Discrete. Continuous Dynamical Systems 2015; 36(4): 1905-1926.

14. J. Fontaine. Une équation semi-linéaire des ondes sur $\mathbb{H}^{3}$, C. R. Acad. Sci. Paris Sér. I Math. 1994; 319: 935-948.

15. K. Fujiwara, A note for the global nonexistence of semirelativistic equations with nongauge invariant power type nonlinearity. Mathematical Methods in the Applied Sciences 2018; 41(13): 4955-4966. 
16. A. Z. Fino, I. Dannawi, M. Kirane, Blow-up of solutions for Semilinear Fractional Schrödinger equations, J. Integral Equations \& Appl. 2018; 30(1): 67-80.

17. A. Z. Fino, I. Dannawi, M. Kirane, Erratum to " Blow-up of solutions for Semilinear Fractional Schrödinger equations " to appear in J. Integral Equations \& Appl.

18. J. Ginibre, T. Ozawa and G. Velo. On the existence of the wave operators for a class of nonlinear Schrödinger equations. Ann. Inst. H. Poincar Phys. Thor. 1994; 60(2): 211-239.

19. J. Ginibre, N. Hayashi. Almost global existence of small solutions to quadratic nonlinear Schrödinger equations in three space dimensions, Math. Z. 1995; 219(1): 119-140.

20. P. Germain, N. Masmoudi and J. Shatah. Global solutions for 3D quadratic Schrödinger equations, Int. Math. Res. Not. 2009; 3: 414-432.

21. P. Germain, N. Masmoudi and J. Shatah. Global solutions for 2D quadratic Schrödinger equations, J. Math. Pures Appl. 2012; 97: 505-543.

22. L. Grafakos, S. Oh, The Kato-Ponce inequality. Commun. Part. Diff. Equ. 2014; 39(6): 11281157.

23. A. Grigoryan, Heat Kernel and Analysis on Manifolds. Ams/ip Studies in Advanced Mathematics, vol.482, 2009.

24. B. Guo, Y. Han and J. Xin. Existence of the global smooth solution to the period boundary value problem of fractional nonlinear Schrödinger equation, Appl. Math. Comput. 2018; 204: 468-477.

25. B. Guo, Z. Huo. Global Well-Posedness for the Fractional Nonlinear Schrödinger Equation, Comm. Partial Differ. Equ. 2011; 36: 247-255.

26. Q. Guo, S. H. Zhu. Sharp criteria of scattering for the fractional NLS, arXiv: 1706.02549

27. Z. Guo, Y. Sire, Y. Wang and L. Zhao. On the energy-critical fractional Schrödinger equation in the radial case, arXiv: 1310.6816

28. N. Hayashi, P. Naumkin. Asymptotics for large time of solutions to the nonlinear Schrödinger and Hartree equations, Amer. J. Math 1998; 120(2): 369-389.

29. N. Hayashi, P. Naumkin. On the quadratic nonlinear Schrödinger equation in three dimensions, Int. Math. Res. Not. 2000; 3: 115-132.

30. Hörmander, Lars. The Analysis of Linear Partial Differential Operators I. Springer Berlin Heidelberg, 2007.

31. M. Ikeda, T. Inui. Small data blow-up of $L^{2}$ or $H^{1}$ solution for the semilinear Schrödinger equation without gauge invariance, J. Evol. Equ. 2015; 15: 571-581.

32. A. Ionescu, G. Staffilani. Semilinear Schrödinger flows on hyperbolic spaces: scattering in $H^{1}$, Math. Ann. 2009; 345: 133-158.

33. A. Ionescu, B. Pausader and G.Staffilani. On the global well-posedness of energy-critical Schrödinger equations in curved spaces.

34. A. Ionescu, F. Pusateri. Nonlinear fractional Schrödinger equations in one dimension, J. Funct. Anal.2014; 266: 139-176.

35. Y. Kawahara. Global existence and asymptotic behavior of small solutions to nonlinear Schrödinger equations in 3D, Differential Integral Equations 2005; 18(2): 169-194.

36. S, Klainerman, G. Ponce. Global,small amplitude solutions to nonlinear evolution equations, Communications on Pure and Applied Mathematics 1983; 21(1): 133-141.

37. N. Laskin. Fractional Schrödinger equation. Phys. Rev. E 2002; 66(5): 249-264.

38. T. Li, Y. Chen. Global solutions for nonlinear evolution equations, Longman Scientific Technical Press, New York, 1991.

39. P. Li, S-T. Yau: On the parabolic kernel of the Schrödinger operator. Acta Math. 1986; 156: 153-201.

40. C. Miao, B. Yuan, B. Zhang, Well-posedness of the Cauchy problem for the fractional power dissipative equations, Nonlinear Anal. 2008; 68: 461-484.

41. H.P. Mckean, J. Shatah. The nonlinear Schrödinger equation and the nonlinear heat equation reduction to linear form, Communications on Pure and Applied Mathematics 1991; Vol. XLIV: 1067-1080.

42. K. Nakanishi. Asymptotically-free solutions for the short-range nonlinear Schrödinger equation. SIAM J. Math. Anal. 32(6) (2001), 1265-1271.

43. T. Ozawa. Long range scattering for nonlinear Schrödinger equations in one space dimension. Comm. Math. Phys. 1991; 139(3) : 479-493.

44. P. Petersen. Riemannian geometry, Graduate Texts in Mathematics, vol.171. Springer-Verlag, New York, 1994. 
BLOW UP OF FRACTIONAL SCHRÖDINGER EQUATIONS ON MANIFOLDS WITH NONNEGATIVE RICCI CURVATURIS

45. A. Pazy, Semigroups of Linear Operators and Applications to Partial Differential Equations, Applied Mathematical Sciences, vol. 44. Springer, New York, 1983.

46. V. Pierfelice. Weighted Strichartz estimates for the Schrödinger and wave equations on DamekRicci spaces. Math.Z. 2008; 260(2): 377-392.

47. C. Sun, J. Zheng. Scattering below ground state of 3D focusing cubic fractional Schrödinger equation with radial data, arXiv: 1702.03148.

48. W.A. Strauss. Nonlinear scattering theory at low energy. Journal of Functional Analysis 1981; 41(1): 110-133.

49. Y. Tsutsumi, K. Yajima. The asymptotic behavior of nonlinear Schrödinger equations, Bull. Amer. Math. Soc. 1984; 11(1): 186-188.

School of Mathematics and Statistics, Changsha University of Science and Technology, Changsha 410114, Peoples Republic of China.

E-mail address: zhlmath@yahoo.com

School of Mathematical Sciences, Sichuan University, Chengdu 610064, Peoples RePUBLIC OF CHINA.

E-mail address: zhaoshiliang@scu.edu.cn 